\title{
Energy-saving Manufacturing Technology for Heating of Injection Stretching Blow Molding Machine
}

\author{
Yi-Chern Hsieh* and Hui-Fang Lin \\ Department of Power Mechanical Engineering, National Formosa University, HuWei 632, YinLin, Taiwan
}

(Received December 1, 2019; accepted May 26, 2020)

Keywords: PET, energy saving, ISBM, CFD

The manufacture of polyethylene terephthalate (PET) containers is now very common, but there has been little research on energy saving in this manufacturing technique. This paper provides a feasible, simple, and low-cost energy-saving manufacturing technology for injection stretching blow molding (ISBM). This study is divided into two parts: an experimental part and a computational part by computational fluid dynamics (CFD). The principle and execution methods of the experiment and computation are delineated in the text. Sensor detection is used to confirm the result of CFD. The finished product produced by the proposed technique has a higher quality than a product made in a factory and the energy saving was approximately $14 \%$. In the future, we will continue to study the integration of various technical details so that machine operators can better understand the methods of energy-saving operation and thereby contribute to environmental protection simultaneously.

\section{Introduction}

Polyethylene terephthalate (PET) bottles are widely used as containers for various beverages including mineral water in our daily lives. These bottles are blown in large quantities via a blow molding machine. Injection stretching blow molding (ISBM) and extrusion blow molding (EBM) are the two main types of blow molding. For ISBM, there are two different techniques of blowing a bottle, which is called a preform or a parison before being blown. One of the techniques is a continuous process involving injection followed by stretch-blowing. The other has two separate processes and is also called the two-stage ISBM process. The two-stage ISBM process can be subdivided into two steps. The first step uses an injection mold to form a preform. The second step of obtaining an initial temperature distribution that reduces energy use is the main target of this research. ${ }^{(1)}$

The performance of the bottle produced by the ISBM process depends on three variables: the initial preform shape, the initial temperature distribution of the preform, and the balance between the stretching and blowing rates. ${ }^{(2)}$ The stress-strain behavior of PET at temperatures above the glass transition temperature depends on the strain rate, strain state, and temperature. ${ }^{(3)}$ The heating conditions will strongly affect the profile of the temperature distribution. The temperature of the preform in the thickness direction or the direction from the neck to the

*Corresponding author: e-mail: ych@nfu.edu.tw

https://doi.org/10.18494/SAM.2020.2780 
bottom will affect the optimal control parameters of the stretching and blowing (kinematic properties). Venkateswaran et al. found that the optical property of anisotropy in the thickness direction is minimum when the temperature of the inner surface of the parison is high. The birefringence of the inner surface in the hoop direction is always higher than that of the outer surface of the bottle regardless of the temperature profile. The bottle surface density correlates with the pearlescence. ${ }^{(4)}$

The temperature distribution of the PET bottle during heating is of great importance, especially for determining the quality of the bottle. ${ }^{(5)}$ In other words, to achieve good blowing results, parameter adjustment during the blowing should be considered together with the initial temperature of the preform. ${ }^{(6)}$

The purpose of this study is to offer a feasible method of finding a set of suitable manufacturing parameters to reduce the energy cost for an ISBM machine. The study of the operational parameters is divided into two parts, namely, the experimental and computational estimation parts. A small infrared heater and an online testing machine are used in the experiment. ${ }^{(7)}$ A calculation of fluid flow as well as a heat transfer analysis make up the computational estimation part. Because different products have different optimal parameters, these parameters are mostly obtained through individual experimental tests. To enable workers to increase their understanding of how energy-saving operating parameters can be found for different products, this paper will describe the experimental test process and results in detail. On the other hand, the reason for the heat flow analysis of the heating box in this paper is to reduce the amount of heat required for heating the parison by utilizing the heat emitted from the infrared lamps and the wall of the heating box. Computational analysis is needed to understand the flow in detail to ascertain how to control the flow angle and air volume of all the fans used to prevent cooling in the heating box so that only a small amount of heat is added to the preform during heating to save energy. Sensors are placed at some specific positions in the box to detect the data to confirm the computational fluid dynamics (CFD) results.

The paper is organized as follows. In Sect. 2, we introduce the experimental part, including the experimental equipment and the purposes of the experiment. Secondly, the computational part is introduced, including the geometry, governing equations, boundary conditions (BCs), and the purposes of the calculation. In Sect. 3, we present and discuss the calculated and experimental results, and conclude the study in Sect. 4.

\section{Experimental and Computational Methods}

The objective of our investigation is to find better energy-saving manufacturing parameters of the CPSB-LSS12 stretch blow machine of ChumPower Company while maintaining the product quality by both experimental and computational methods. In our previous research, ${ }^{(8)}$ the calculation and analysis of the results of infrared heating were carried out and verified by experiments. Here, we provide a computational method to analyze the reused heat flow field in the heating box for preventing the cooling of the heated parison and an experimental method with a real machine to find out how to use the minimum number of infrared lamps and the minimum operating power of each lamp that can meet the product quality requirements. 


\subsection{Experimental part}

The main purpose of the experimental part of this paper is to determine the power of each infrared lamp so that the desired surface temperature distribution of the preform can be achieved. ${ }^{(1,9)}$ The shape, material, and structure of each preform to be blown may be different, so it is necessary to know the optimum temperature distribution of the workpiece before blowing (the optimal temperature distribution needs to be tested, which is another research topic beyond the scope of this article). After that, we can find the most energy-efficient steps for heating preforms. The aim of this experiment is to provide an energy-saving way to heat the preform.

The actual experimental platform is shown in Fig. 1. The infrared camera used is shown on the left-hand side of Fig. 1. It can instantly collect the surface temperature distribution of heated preforms with a precision of $0.01{ }^{\circ} \mathrm{C}$. Infrared lamps, which are the most important heating part of the entire blowing machine, are in the upper right of the figure.

Upon entering the CPSB-LSS12 machine, the preform passes through several heating boxes in the counterclockwise direction, as shown in Fig. 2, where it is heated. After heating, the viscoelastic preform is carried into the stretch-blowing mold to form the final product by the stretching-blowing process. The preforms used in the experiment are shown in Fig. 3. In this paper, if the experimental results of different preforms have the same trend, we will only display the representative results in graphs. Before performing the experiment to determine the thermal properties of all preforms, we tested the temperature properties of heated parisons to confirm the heating efficiency of the lamp and the rotation of the preform.

\subsubsection{Experiment to validate the properties and heating stability of the lamp}

In addition to the calibration needed for the camera itself, we determined whether the property of the infrared lamp matched that in the manual, presented as the change in energy density relative to the space angle $\theta$. From Fig. 4, we found that $C(\theta)[=B(\theta)-A(\theta)]$, the temperature distribution after heating with a $1000 \mathrm{~W}$ lamp, is close to constant, which means

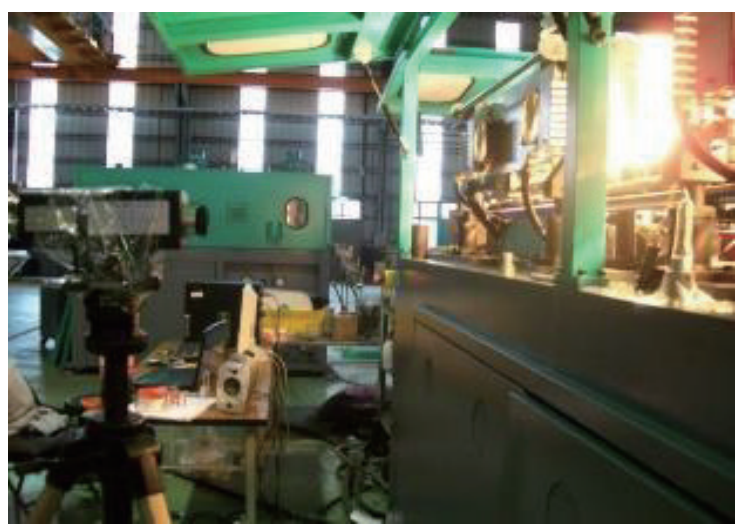

Fig. 1. (Color online) Workplace and infrared camera. 


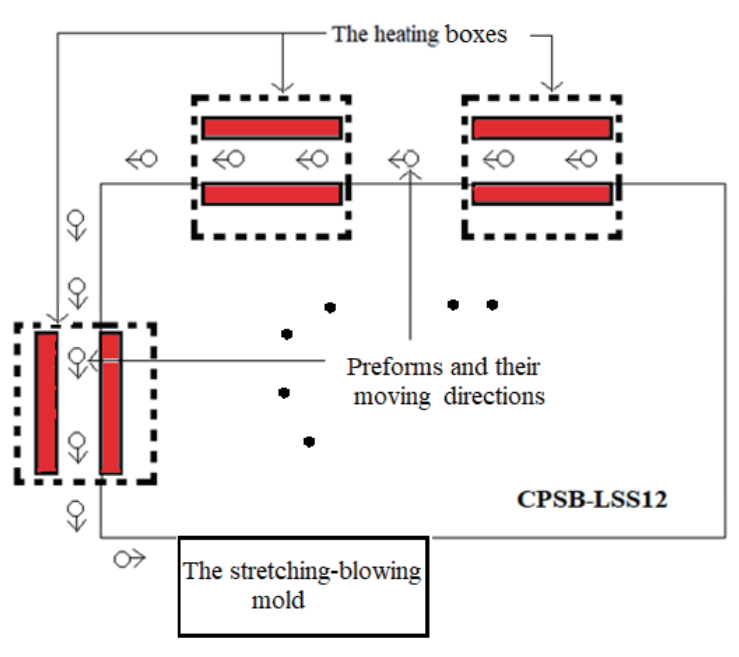

Fig. 2. (Color online) Schematic diagram of top view of CPSB-LSS12.

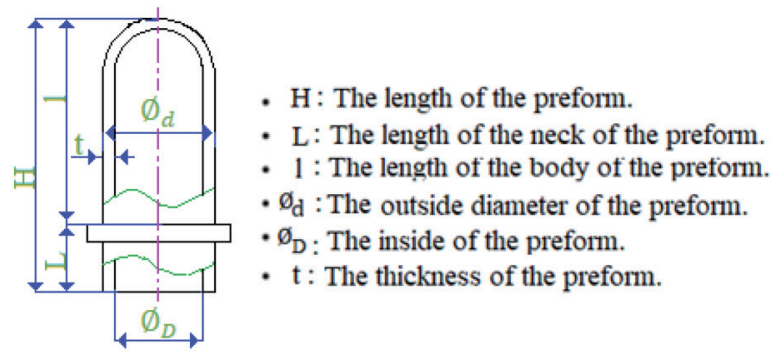

\begin{tabular}{|c|c|c|c|c|c|c|}
\hline Model & $1(\mathrm{~mm})$ & $\emptyset_{\mathrm{d}(\mathrm{mm})}$ & $\emptyset_{\mathrm{D}}(\mathrm{mm})$ & $\mathrm{t}(\mathrm{mm})$ & $\mathrm{L}(\mathrm{mm})$ & $\mathrm{H}(\mathrm{mm})$ \\
\hline 1 & 70.5 & 25.75 & 25.2 & 0.296 & 18.5 & 89 \\
\hline 2 & 105 & 26 & 25 & 0.514 & 18.5 & 123.5 \\
\hline 3 & 129 & 25.4 & 21.7 & 1.856 & 21 & 150 \\
\hline 4 & 72.5 & 25.04 & 22.3 & 1.381 & 18.5 & 91 \\
\hline 5 & 68 & 22.65 & 21.65 & 0.487 & 21 & 89 \\
\hline 6 & 87 & 23 & 21.5 & 0.81 & 18.5 & 105.5 \\
\hline
\end{tabular}

Fig. 3. (Color online) Dimensions of preforms.
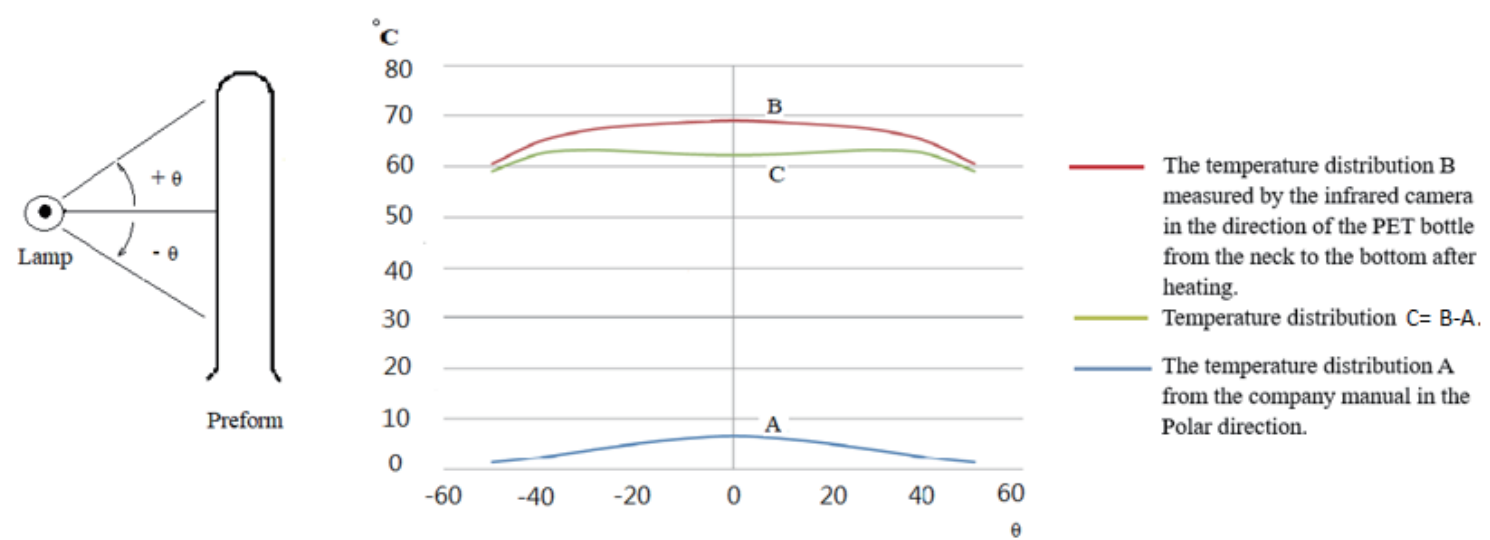

Fig. 4. (Color online) Verification experiment of the radiation heating property.

that the properties of the heating lamp are as expected. From this experiment, we can confirm that the measured temperature is reliable in the direction of the theta. Thus, we can carry out experiments to study the operating power and the position of each tube to achieve the ideal surface temperature distribution of the preform.

In the heating process, since the preform is constantly rotating and advancing, we first investigate whether the rotation of the preform causes uneven heating. To establish this, we performed a corroboration experiment. First, as shown in Fig. 5, we divided the preform into seven sections in the direction of its length, and a horizontal line was drawn on each segment (parallel to the top face of the bottle) as a mark, with the lines $10 \mathrm{~mm}$ apart. All experimental results verified that the mechanical heating system has a uniform heating effect on the preform 

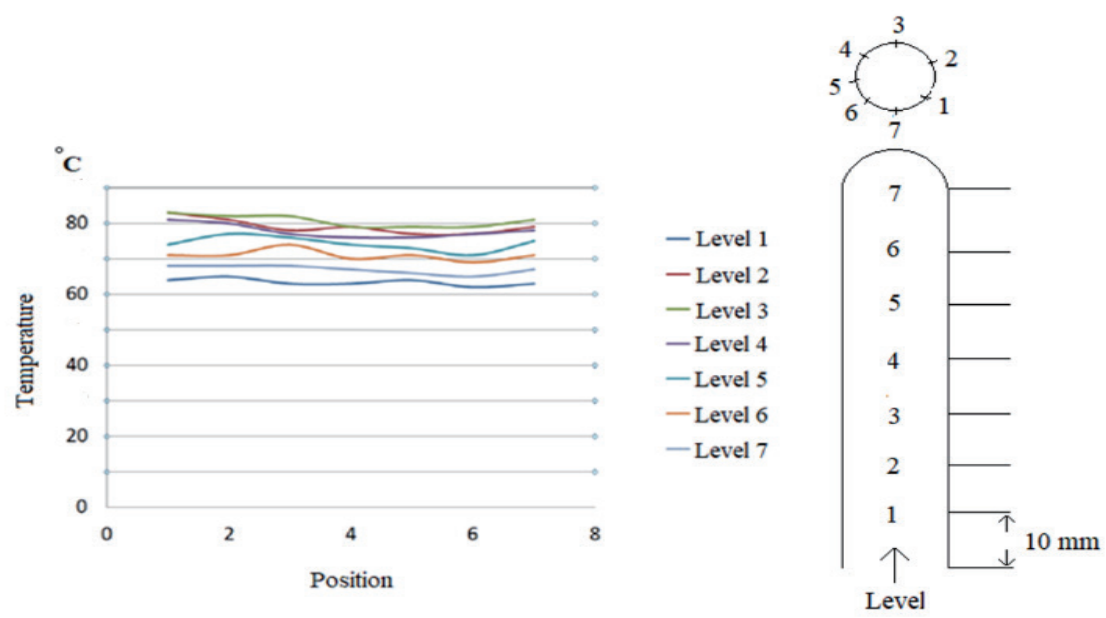

Fig. 5. (Color online) Temperature distribution around circles drawn on different sections on the outer surface of a parison.

under rotation. We also calculated the expanded uncertainty $(99.5 \%)$ for the seven different sections to be $3.62,4.28,5.66,7.32,7.63,8.75$, and 7.05. In addition, the ISBM gives a uniform heating property, which was verified by a statistical test (Mann-Whitney $\mathrm{U}$ test, $p$-value $=0.263$ ); we performed the $U$ test as the underlying distributions of the data may not be Gaussian. In other words, the spatial distribution of the heating efficiency of the lamp and the effect of the rotation of the preform on the heating efficiency were confirmed to be as expected.

\subsubsection{Physical quantities measured by infrared camera and sensors}

Before the preform is fed into the blowing model, the surface temperature of the preform must be increased to a satisfactory level during infrared heating. To obtain the surface temperature distribution of the heated preform, we used the infrared camera to capture images of temperature distributions. Each of the preforms was divided into eleven regions for detecting the surface temperature by the camera as shown in Fig. 6(a). We considered the temperature distributions in Figs. 6(b) and 6(c) under different physical conditions to analyze the appropriate production operating parameters with real data. We also used platinum-type sensors, which can measure temperatures up to $850{ }^{\circ} \mathrm{C}$, to measure the heating box temperature at some specific positions. Although we measured the temperature distribution inside and outside the preform, computations were still required to make up for the shortcomings.

\subsection{Computational part}

To reduce the heating power by reusing the dissipated heat from lamps or the box, a threedimensional (3D) CFD model is needed to calculate the flow field in the heating box in detail. The purpose of this computation is to understand the flow of the wind blown by the exhaust heat in the heating chamber and to find a better flow field so that the shape of the surface temperature distribution of the preform can be maintained. As shown in Fig. 7, there is a row 


\begin{tabular}{llll}
\hline Region & Position $(\mathrm{mm})$ & Region & Position $(\mathrm{mm})$ \\
\hline 1 & 5 & 7 & 35 \\
2 & 10 & 8 & 40 \\
3 & 15 & 9 & 45 \\
4 & 20 & 10 & 50 \\
5 & 25 & 11 & 55 \\
6 & 30 & &
\end{tabular}

(a)

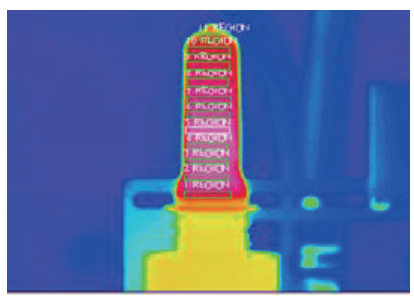

(b)

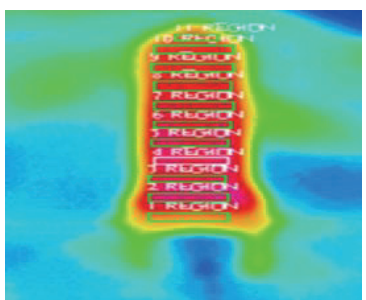

(c)

Fig. 6. (Color online) Marked region (a) and temperature distributions detected by the infrared camera (b) and (c). (a) Distance from preform neck to bottom of the preform. (b) Temperature of inner surface of the heated preform. (c) Temperature of outer surface of the heated preform.

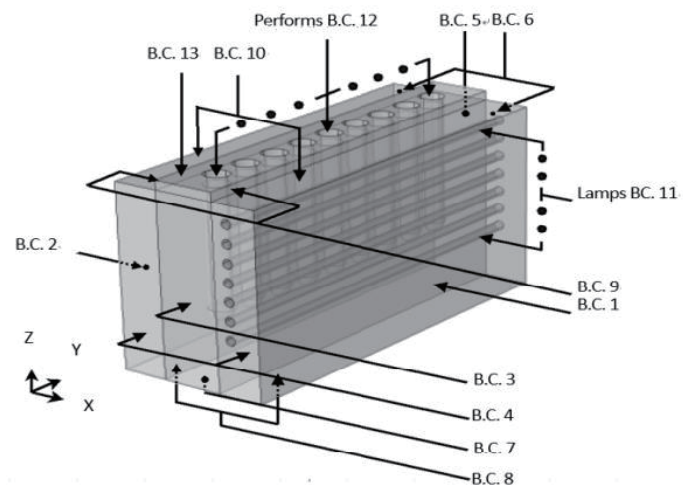

(a)

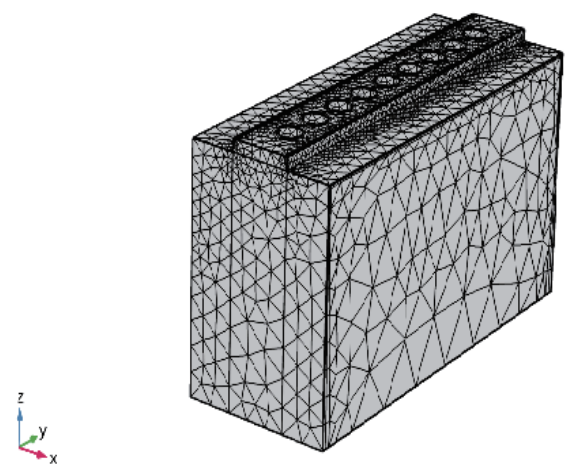

(b)

Fig. 7. (Color online) Schematic of (a) heating box with various BCs and (b) initial mesh.

of preforms in the heating box, which are arranged according to their geometrical dimensions. The flow of the fluid in the heating chamber is an incompressible unsteady viscous laminar flow. The governing equations are

$$
\begin{gathered}
\nabla \cdot \boldsymbol{u}=0, \\
\rho \frac{\partial \boldsymbol{u}}{\partial t}+\rho \boldsymbol{u} \cdot \nabla \boldsymbol{u}=-\nabla p+\nabla \cdot \tau+\rho \boldsymbol{g}, \\
\rho C_{p} \frac{\partial T}{\partial t}+\rho C_{p} \boldsymbol{u} \cdot \nabla T-\nabla \cdot \boldsymbol{q}=Q,
\end{gathered}
$$

where $\boldsymbol{u}$ : velocity vector, $p$ : air pressure, $T$ : air temperature, $\boldsymbol{\tau}$ : shear vector, g: gravity, $Q$ : irradiative heat flux density, and $q=-k \nabla T$ ( $k$ : heat transfer coefficient).

All BCs are shown in Fig. 7. BCs 1 and 2 are for the two walls of the heating box with a Dirichlet-type boundary $|\boldsymbol{u}|=u_{0}$ and $T=T_{0}$. Both sides have wind blowing into the box to prevent cooling. BCs 3,5 , and 13 are open boundaries $\left(P=P_{0}\right)$ of the corridor along which the 
preforms pass during processing. $\mathrm{BCs} 4,6,8$, and 10 are for the walls of the heating box with the no-slip condition. The flow that blows up (or sucks down) from below is from $\mathrm{BC} 7$, where $|\boldsymbol{u}|=v_{0} . \mathrm{BC} 9\left(T=T_{1}\right)$ is the temperature of the hot air applied near the neck of the preforms, and the hot air flow is from the back of the heat box. ${ }^{(7)}$ The BC 11 of the lamps is the constant operation temperature $T=T_{2}$. The BC 12 of the parisons is the operating speed $\omega=\omega_{0}$. The computation in this paper is compared with the results of Hsieh et al. ; $^{(10)}$ however, in this paper, the model conditions of unsteady flow, all preforms undergoing rotation, and the infrared heating speed are all taken into account to make the computation model closer to the actual situation.

The mathematical model is set up as above. The number of degrees of freedom of the final mesh is 2903200 and the average $y+$ value obtained over the preforms is 0.28 . The computing time step during time marching is $10^{-3} \mathrm{~s}$ and the total heating time depends on the number of heating boxes and the moving speed of the parisons. The results obtained from the software can clearly identify the wind flow rate and flow direction required to improve the temperature distribution profile.

\section{Results and Discussion}

The factors affecting the product quality (excluding the type of preform material and the process parameters of the blow molding) include the power of the lamp, the heating time, the temperature distribution, the lamp spacing, the distance between the lamp and the preform, the infrared reflector, the way the fans are used to prevent cooling, the environmental cooling effect, the preform thickness, the preform position, and the movement and rotation speeds of the preform. Except for the fans and the environmental cooling effect, all these factors are related to the heating process. Among these factors, some are independent, while some are related.

\subsection{Experimental results}

When the geometry of the preform, the manufacture production speed (determined by the production volume and completion time), and the temperature distribution [which requires another experiment ${ }^{(1)}$ ] of the heated preform are chosen, the following experiments are sequentially performed in steps. We first determine the power of each lamp, the distance between the infrared tubes when heating, and the distance between the tube and the preform. Since the distance between the tube and the preform and the distance between tubes are adjustable, the experimental values are easily obtained. Secondly, we examine the effect of each lamp power. Among the experimental results, we show only representative images below owing to the limited space.

\subsubsection{Experiment to determine parameters of lamps and rotation speed of preforms}

To analyze the combined effect of the power of each lamp on the heating effect of the preform, it is necessary to understand the heating effect on a tube. The effect of a single lamp 
on the surface heating of the preform with the same distance between the preform and the lamp and the same heating time for different power intensities is shown in Fig. 8. The position means the distance from the tip to the tail of the preform. This figure shows the extent to which different powers $(1000-3000 \mathrm{~W})$ of a lamp can affect the range in which the preform is warmed. There is also a similar trend for preforms with other thicknesses.

As shown in Fig. 9, we found that heating results measured in the experiment with three lamps simultaneously operating were almost identical to those obtained by the addition of the results for the three individual lamps shown in Fig. 8. Moreover, when we compared the presence or absence of the fan and the rotation of the preform during heating, we found that the two factors, the fan and rotation, do not affect the addition property for heating. This result is very helpful for experimentally estimating or adjusting the operation parameters of all lamps used to heat the preform in an energy-saving manner. As shown in Fig. 10, we chose two different distances between the lamps to measure the temperature distribution of the heated preform. In an environment with the same operation conditions, a distance of $6 \mathrm{~mm}$ is better than that of $8 \mathrm{~mm}$. From all the above experiments, we can choose the power of the lamps used for heating.

For the M1 preform model, we found that the CPSB-LSS12 machine only needs to turn on five lamps with powers of L1 $=2520 \mathrm{~W}, \mathrm{~L} 2=2700 \mathrm{~W}, \mathrm{~L} 3=1200 \mathrm{~W}, \mathrm{~L} 4=1300 \mathrm{~W}$, and

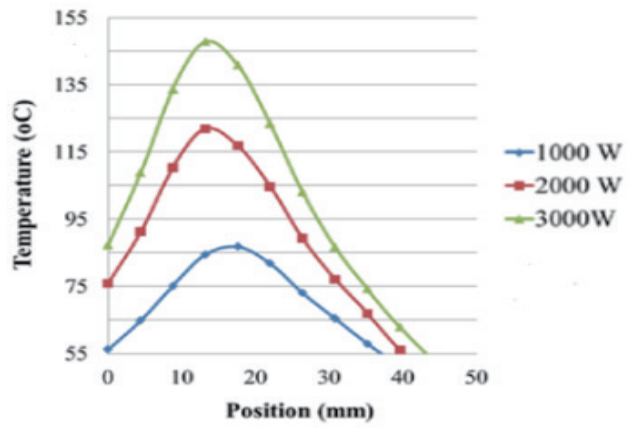

Fig. 8. (Color online) Effect of the lamp power on the heating effect of the preform.

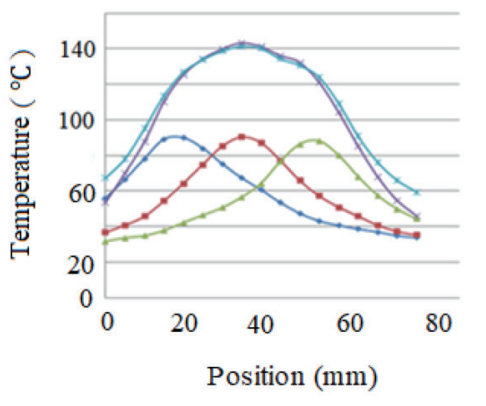

(a)

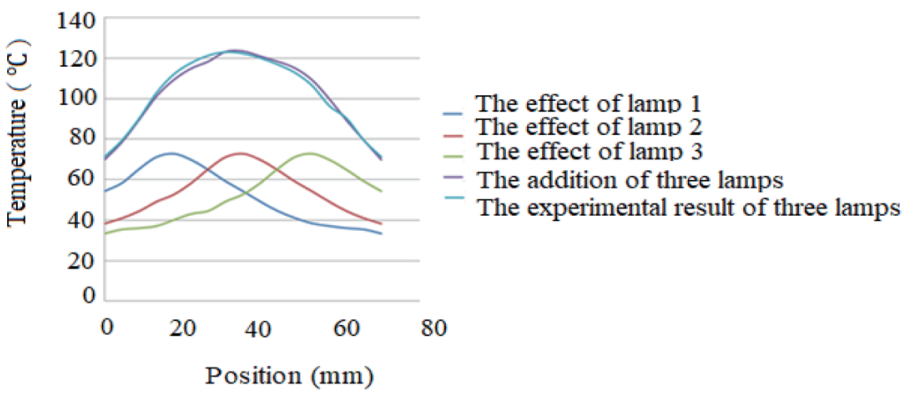

(b)

Fig. 9. (Color online) Results of multiple lamps predicted by the superposition of results for individual lamps. (a) Without the fan or preform rotation. (b) With the fan and preform rotation. 


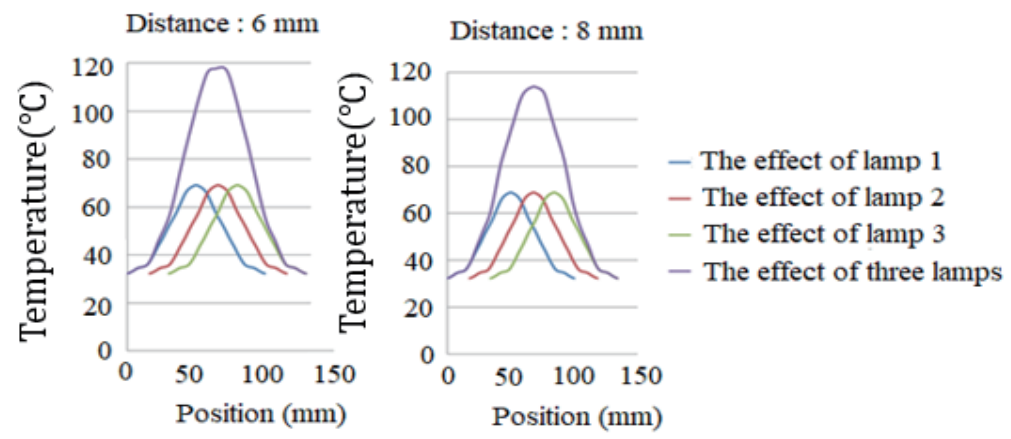

Fig. 10. (Color online) Heating effect by adjusting the spacing of lamps according to the machine scale.

$\mathrm{L} 5=1900 \mathrm{~W}$ to obtain the ideal surface temperature distribution of the preform instead of turning all seven lamps on. The experimentally determined rotation speeds of the preform of $20-80$ on the scale of the machine are shown in Fig. 11. The speed of 60 gives the distribution closest to the ideal temperature distribution for model M1. The other models show a similar trend in terms of the rotation speed. The various processing parameters discussed above are related to the preform heating quality. Next, we will discuss issues related to the temperature drop on the preform surface.

\subsubsection{Fan and environmental effects}

Both the fan and the environment will affect the surface temperature of heated preforms. As shown in Fig. 12, it was found that under optimized heating conditions, the fan power of 20 on the machine's scale most effectively prevented the cooling of the preform surface. This result is useful for the computation model operating under its BCs to calculate the best preform flow field to prevent surface cooling in the heating chamber.

\subsection{Computation results and validation}

The surrounding environment decreases the surface temperature of the heated preform as it passes through the space between any two heating boxes. The temperature of the space is that of the surrounding room, and the heated preforms are cooled under this condition. Thus, we have to measure the amount of dissipated heat from the preforms at room temperature.

The cooling by the surrounding environment of all six preforms is tested. The results in Figs. 13(a)-13(c) are for three preforms (M1, M2, and M4). If the preform thickness is less than $2.5 \mathrm{~mm}$, as long as the heat dissipation time is $7-8 \mathrm{~s}$, the inner temperature of the preform is higher than the outer temperature. However, if the thickness is greater than $2.5 \mathrm{~mm}$, the inner temperature is lower. We also found that the surface temperature of the preform decreases by about $1-1.5^{\circ} \mathrm{C} / \mathrm{s}$ owing to cooling by the surrounding environment. Experiments of the heating and prevention of preform cooing have been explained, and we will present the calculations carried out to find a better flow field in the next section. 


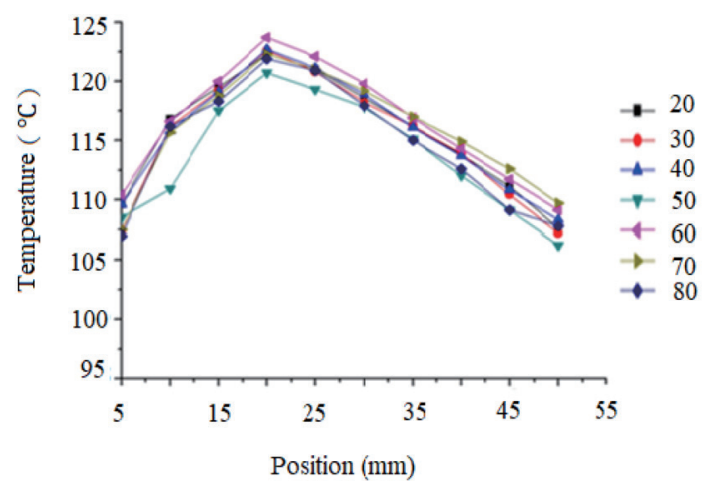

Fig. 11. (Color online) Influence of the rotation speed of parison M1 on the heating effect.

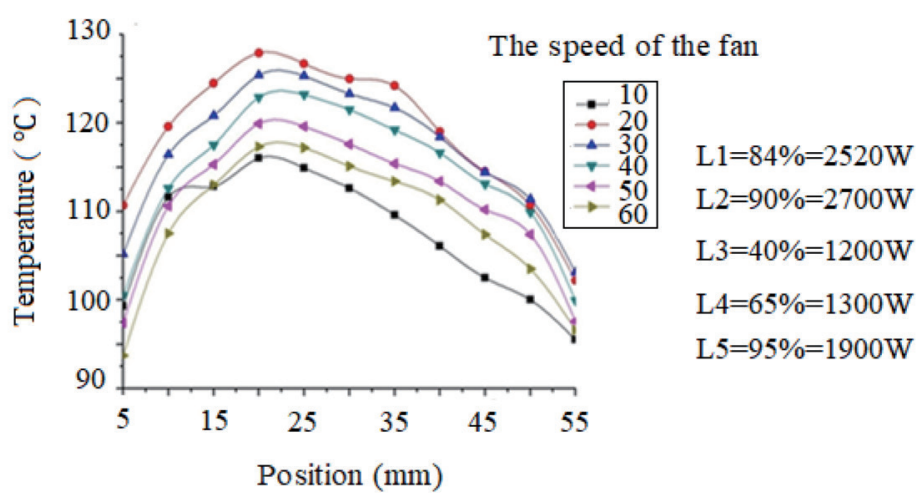

Fig. 12. (Color online) Effect of magnitude of the power of the fan for preventing cooling on temperature distribution.

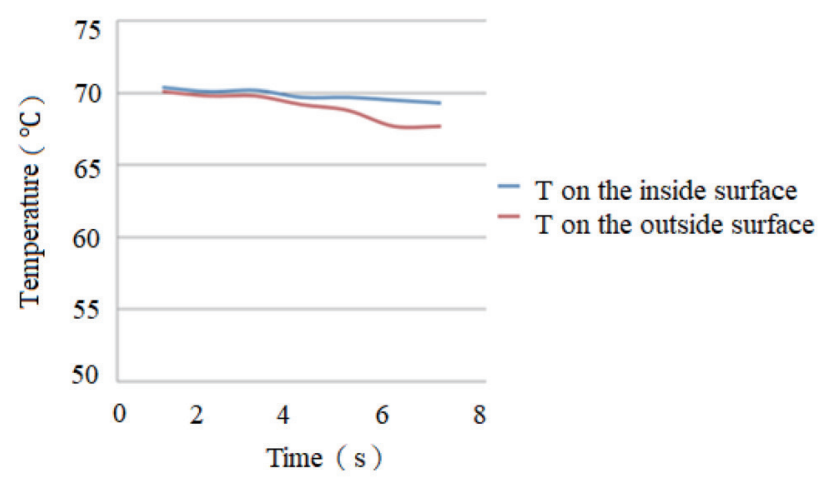

(a)

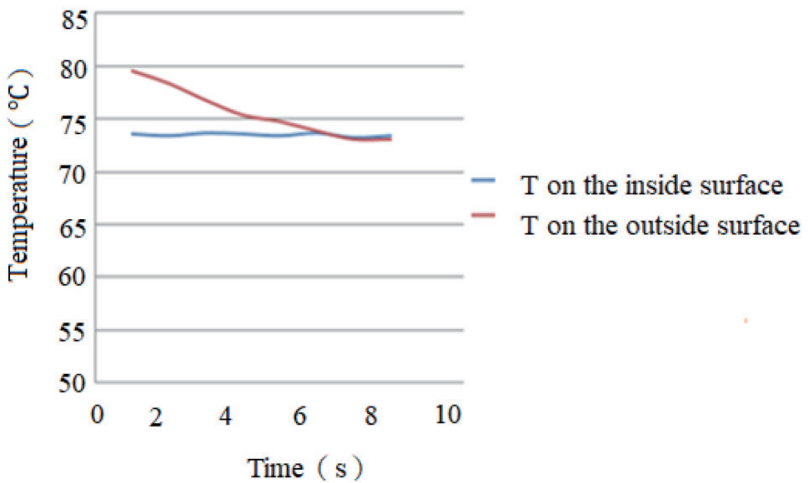

(b)

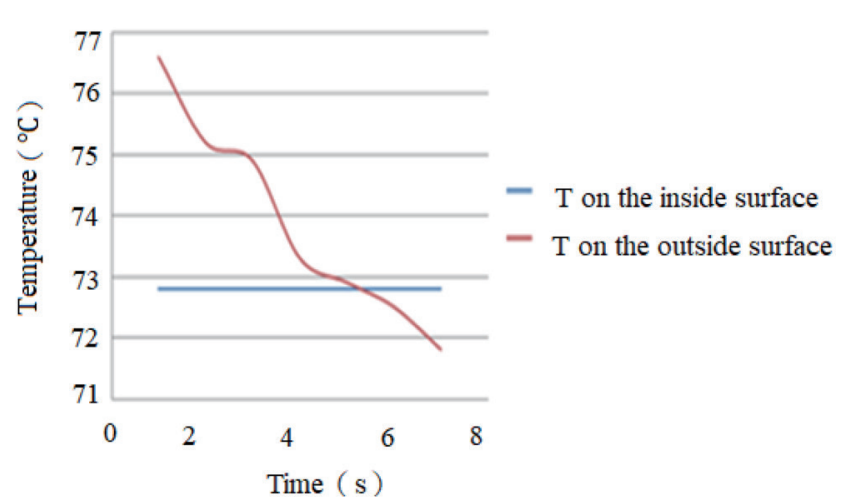

(c)

Fig. 13. (Color online) Time-dependent temperatures of different parisons during cooling to room temperature.

Although our engineering calculations through theoretical modeling are slightly different from the actual situation, we can at least find the best flow field. Because the optimization of the airflow field in the heating box ameliorates the efficiency of preventing the cooling of the preform, CFD is used to estimate the direction in which the fan should blow. To calculate 
the results as accurately as possible, increasing the accuracy of the simulated preform heating process is the fundamental goal of the calculation. This also means that the preform heating rate must be as close as possible to the experimental results obtained during the simulated heating process. As can be seen from Fig. 14(a), the heating rate in this calculation is similar to that in the experimental results shown in Fig. 15.

During heating, the preforms move from left to right as shown in Fig. 14(b). The fans used to prevent cooling blow from both the left and right sides to the center region of the box as in Fig. 14(c) and from the bottom side of the plot in Fig. 14(b) to the $+Z$ direction. The volume of the room-temperature air blown upwardly by the fan from the bottom is greater than that of the hot air blown from the left and right sides. The computed flow field around each of the parisons has vortexes as shown in Fig. 14(c). This phenomenon revealed that because of the rotation of the preform, the flow field is different from the results for the no-slip condition in Ref. 10. From Table 1, we find that some of the convective heat flux is negative, which means that during the heating of the preform, the heat preservation effect is achieved by using the reused hot gas to maintain a moderate degree of heating. $\mathrm{UH}$ or $\mathrm{HH}$ is the best choice from the standpoint of maintaining the warming effect.

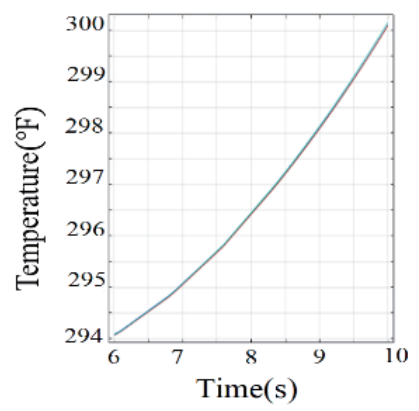

(a)

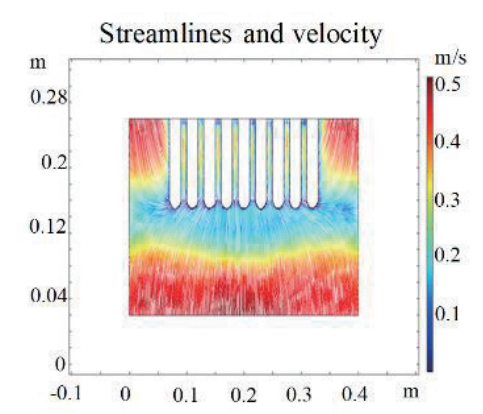

(b)

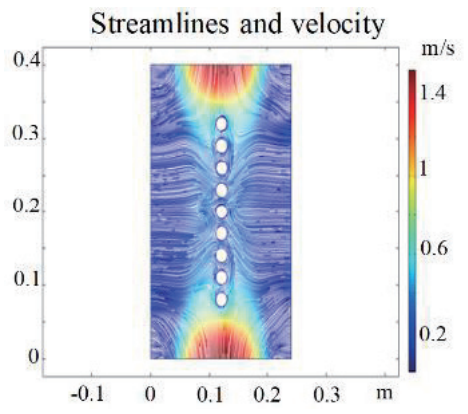

(c)

Fig. 14. (Color online) (a) Heating rate obtained by computation; (b), (c) flow velocity field in the heating box: $+X$ and $-Z$ views, respectively.

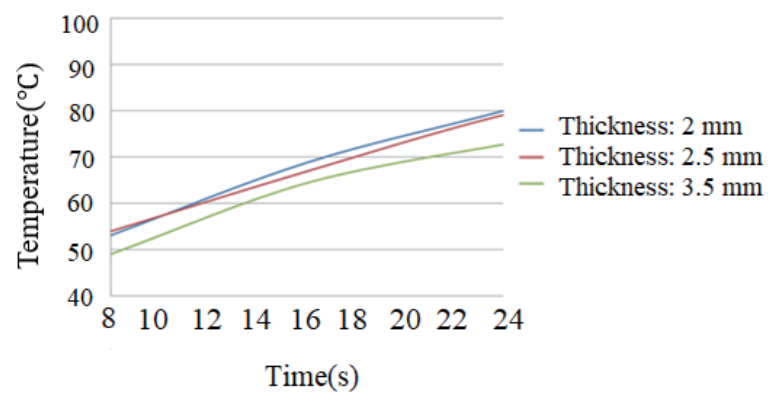

Fig. 15. (Color online) Heating rates for different thicknesses of parisons under the same heating conditions. 
Table 1

Different blowing angles of fans under BCs 1 and 2.

\begin{tabular}{ccccccccc}
\hline UU & UD & UH & DU & DD & DH & HU & HD & HH \\
\hline-0.11 & 0.0273 & -0.07 & 0.0001 & 0.033 & -0.0049 & -0.0097 & 0.0303 & -0.0698 \\
\hline
\end{tabular}

$\mathrm{U}$ represents the blowing direction with an elevation angle of $45^{\circ}$ from the $x y$ plane.

D represents the blowing direction with a depression angle of $45^{\circ}$ from the $x y$ plane.

$\mathrm{H}$ represents the horizontal blowing direction.

The first capital letter represents the wind blowing angle under BC 1 , and the second letter represents the angle under BC 2 . The numbers in the table represent the time-averaged convection heat flux $\left(\mathrm{W} / \mathrm{m}^{2}\right)$ on the surface of the preform.

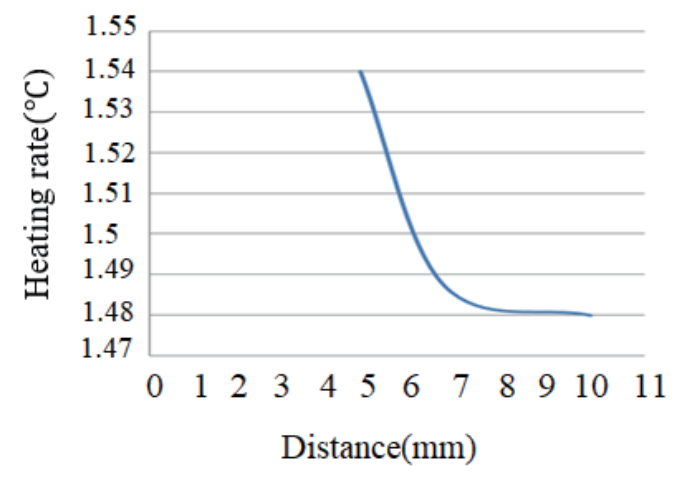

Fig. 16. (Color online) Average heating rate versus the distance between the lamp and the preform.

We tested the capability of each preform (M1-M6) to absorb heat and found that the heating rate of the preform was nearly constant at a fixed distance between the preform and the tube, as shown in Fig. 15. In addition, we also need to determine the relationship between the heating rate and the distance between the preform and the tube. As shown in Fig. 16, we take the preform to be produced as an example, and the relationship between the heating rate and the distance is summarized under the energy-saving operating conditions. From Table 1, there are nine different ways in which the recovered hot air is blown into the heating box, and both UH and $\mathrm{HH}$ are considered as the two best energy-saving ways for manufacturing.

The nine different temperature distributions in Fig. 17 are obtained with different blowing angles of the fans to prevent cooling. Because of the different flow fields, the surface temperature distributions of the preforms differed despite the heating conditions being exactly the same. The inner wall temperature of the preform is slightly lower than the outer wall temperature, which means that even though the external wall temperature of the preform is lowered by the effect of the ambient temperature, the hot returned gas mixed in the heating box can keep warming the preform. The insulation effect maintains the temperature. The different flow fields caused the temperature distribution to change slightly. Although the reused hot air can increase the heating effect, it still keeps the surface temperature distribution curve similar to that obtained with the infrared lamps in the experimental stage.

In Fig. 18, we compare our experimental results with those of Bordival et al., ${ }^{(1)}$ and the temperature distribution on the surface of the preform of our cases is close to that of 

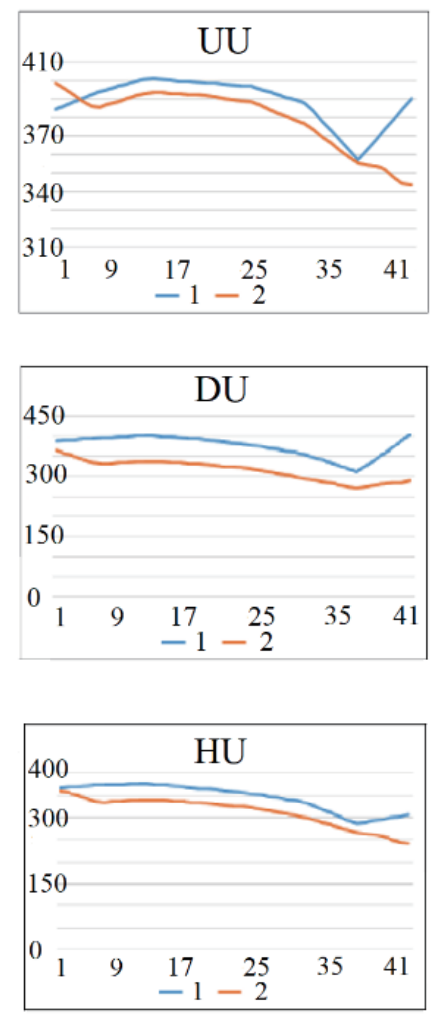
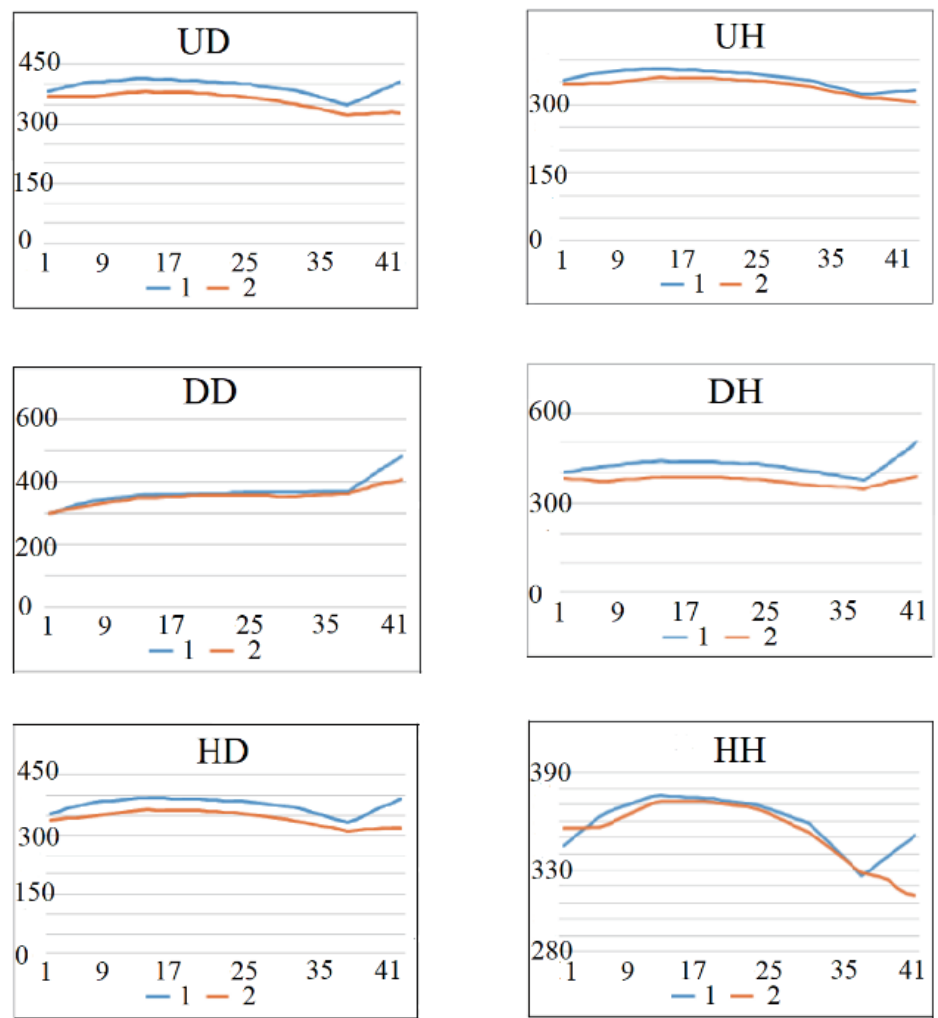

Fig. 17. (Color online) Temperature distributions on the outer and inner preform surfaces. The $X$ axis represents horizontal positions from the neck to the bottom of the preform. The $Y$ axis is the temperature in $\mathrm{K}$. Line 1 is the temperature distribution on the outer wall of the preform and Line 2 is that on the inner wall. The heating time is $20 \mathrm{~s}$ and the power is the same for all nine cases.

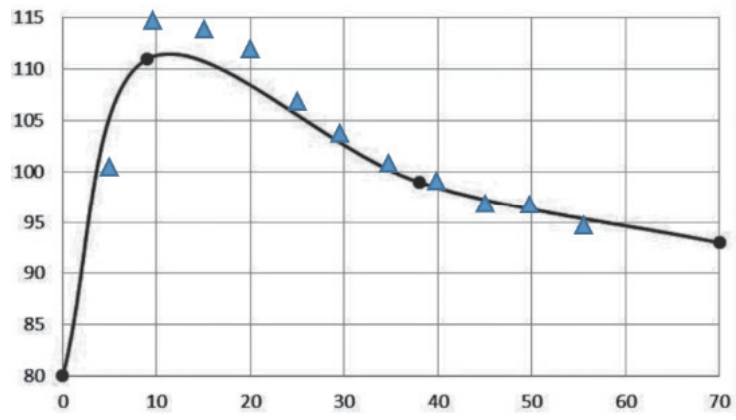

Fig. 18. (Color online) Comparison of our experimental results with those of Bordival et al. ${ }^{(1)}$

Bordival et al. The trend of the temperature distribution is similar, and the product thickness is also verified to be uniform. This will be discussed in the next section. In this energysaving operation, we control the preform tail temperature to be slightly lower than that of Bordival et al. because we can use the recycled hot air to compensate for the missing part. 
In addition, it can be seen from Fig. 17 that the temperature at the bottom of the preform increases with the heating time. This phenomenon indicates that the air blown up from the bottom of the heating box will mix with the hot air from both sides and be blown to the bottom of the preform, as shown in Fig. 19, so the temperature of the preform will increase owing to the hot gas. Therefore, a flow field with excessive hot air will make the temperature higher than the ideal temperature. It can be seen in Fig. 19 that the bottom of the preform and the neck have direct contact with the hot gas having a relatively high temperature, resulting in a high temperature at the neck and bottom.

\subsection{Actual implementation and verification}

To confirm that the computation result of the temperature distribution in the heating box is reliable, we set temperature sensors at four specific positions: $\mathrm{P}(0,0.2,0.23), \mathrm{Q}(0.24,0.2,0.23)$, $\mathrm{R}(0,0.35,0.23)$, and $\mathrm{S}(0.24,0.35,0.23)$ (unit of $\mathrm{m})$. Because the hot air is rising, we chose these four positions to measure the temperature for comparison with the CFD result. The results are shown in Table 2. All data are averaged over ten measurements under a steady-state condition. According to Table 2, the CFD results are close to the measurement results.

When all the operating parameters are found, the final practical verification experiments will be held to confirm that the research results are satisfied. We use the company's manufacturing parameters based on their experience to blow 300 bottles, and we also blow 300 bottles with the parameters found in this study. The statistical data are shown in Table 3, where 'company' means the manufacturing parameters from company experience and 'experiment' means the parameters from our research result. As shown in Fig. 20, the average thickness distribution of the bottles blown with the company's parameters is relatively poor. In addition, the total power

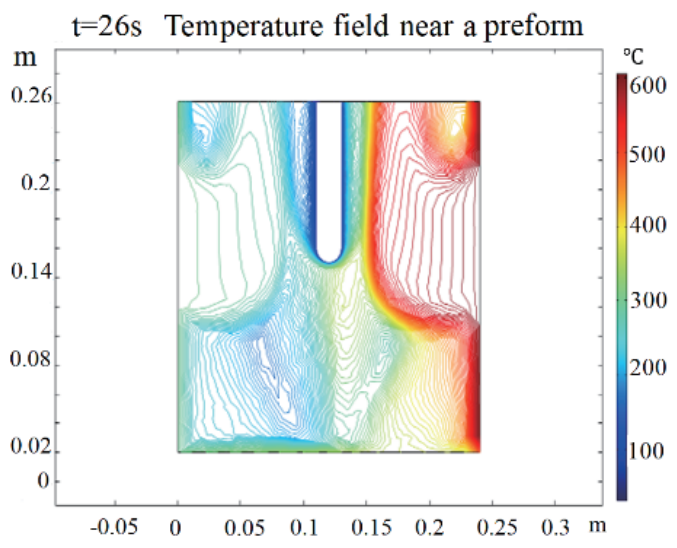

Fig. 19. (Color online) Temperature $\left({ }^{\circ} \mathrm{C}\right)$ distribution around the parisons region.

Table 2

Verification of computation results with experimental data.

\begin{tabular}{lcccc}
\hline & $\mathrm{P}$ & $\mathrm{Q}$ & $\mathrm{R}$ & $\mathrm{S}$ \\
\hline Computation $\left({ }^{\circ} \mathrm{C}\right)$ & 257 & 585 & 248 & 572 \\
Experiment $\left({ }^{\circ} \mathrm{C}\right)$ & 249 & 591 & 252 & 580 \\
\hline
\end{tabular}


Table 3

Statistics of thicknesses (mm) in Fig. 20.

\begin{tabular}{lllllllllllll}
\hline \multirow{2}{*}{ Company } & Avg. & 1.015 & 0.445 & 0.390 & 0.425 & 0.63 & 0.725 & 0.870 & 1.085 & 0.975 & 0.960 & 0.850 \\
\cline { 2 - 11 } & S.D. & 0.021 & 0.027 & 0.012 & 0.011 & 0.01 & 0.018 & 0.021 & 0.026 & 0.028 & 0.019 & 0.031 \\
\hline \multirow{2}{*}{ Experiment } & Avg. & 0.6 & 0.44 & 0.39 & 0.42 & 0.45 & 0.44 & 0.425 & 0.5 & 0.7 & 0.96 & 1.14 \\
\cline { 2 - 11 } & S.D. & 0.019 & 0.011 & 0.011 & 0.010 & 0.015 & 0.009 & 0.016 & 0.018 & 0.021 & 0.019 & 0.016 \\
\hline
\end{tabular}

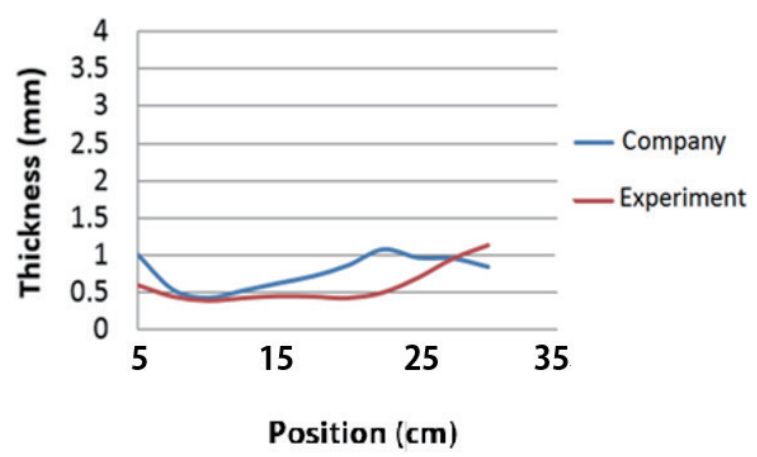

Fig. 20. (Color online) Results of verification experiment for average bottle thickness for two different ways of producing products.

depletion from the main energy consumption under our operating parameters is $9250 \mathrm{~W}$, while that for the company's parameters is $10800 \mathrm{~W}$. Because of the manufacturing method used in this study, the energy required by the fans is slightly higher than that used with the company's parameters. The energy consumed by the lamps, however, is about $14 \%$ less than that used with the company's parameters.

\section{Conclusions}

This study provides a low-cost, simple, and feasible method for the energy-efficient manufacture of PET bottles. The study is divided into experimental and computational parts. The purpose of the experimental part is to find suitable energy-saving manufacturing parameters including the power of each infrared tube, the distance between the lamps, the distance between the preform and the lamp, the rotation speed of the preform, and the strength of the fans. The purpose of the computational part is to determine how to most effectively return the heat released from the heating box to the heating chamber to prevent the change in preform surface temperature distribution by environmental cooling. The results of the study show that the final product has a better thickness distribution than a product produced by the original manufacturing method, both for the body of the bottle and its bottom. This result is partly due to sensor detection results, which verified the CFD results. In conventional manufacturing, seven lamps must be used simultaneously. However, only five lamps are required using the parameters found in this study, reducing energy use by about $14 \%$. The method of obtaining improved parameters in the manufacturing procedure can be provided to bottle makers to reduce the energy required to manufacture the preform and the finished product. 


\section{Acknowledgments}

This work was supported by the ChumPower Company. We thank ChumPower and all our students, Pham Thi Thanh Hoi, Doan Minh Hai, and especially Nguyen Dinh Nghia, who helped us in the experimental work.

\section{References}

1 M. Bordival, F. M. Schmidt, Y. L. Maoult, and V. Velay: Polym. Eng. Sci. 49 (2009) 783. https://doi. org/10.1002/pen.21296

2 F. M. Schmidt, J. F. Agassant, and M. Bellet: Polym. Eng. Sci. 38 (1998) 1399. https://doi.org/10.1002/ pen. 10310

3 M. C. Boyce, S. Boyce, P. G. Socrate, and Llana: Polymer 41 (2000) 2183. https://doi.org/10.1016/S00323861(99)00406-1

4 G. Venkateswaran, M. R. Cameron, and S. A. Jabarin: Adv. Polym. Tech. 17 (1998) 237. https://doi.org/10.1002/ (SICI)1098-2329(199823)

5 Y. M. Luo, L. Chevalier, F. Utheza, and X. Nicolas: Int. Polym. Proc. 30 (2015) 554. https://doi. org/10.3139/217.3092

6 L. Chevalier, C. Linhone, and G. Regnier: Plast. Rubber Compos. 28 (1999) 393. https://doi.org/10.1179/146580 199101540556.

7 Y.-C. Hsieh and M. H. Doan: Int. J. Adv. Manuf. Technol. 98 (2018) 2357. https://doi.org/10.1007/s00170-0182220-6

8 Y.-C. Hsieh, M. H. Doan, and T. T. Pham: Adv. Mater. Res. 750 (2012) 591. https://doi.org/10.4028.591-593.750

9 Z. J. Yang, E. Harkin-Jones, G. H. Menary, and C. G. Armstrong: J. Mater. Process. Technol. 120 (2004) 153. https://doi.org/10.1016/j.jmatprotec.2004.04.203

10 Y.-C. Hsieh and H.-F. Lin: Proc. IEEE Int. Conf. Advanced Manufacturing 216 (2018) 16. https://doi. org/10.1109/AMCON.2018.861508

\section{About the Authors}

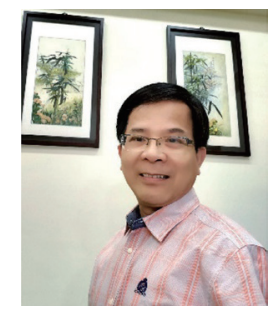

Yi-Chern Hsieh received his Ph.D. degree from Yun-Lin Institute of Technology, ROC, in 2009. He has been engaged in the application of a variety of adaptive scientific computation methods in various engineering problems. At present, he is focusing on the fields of medical engineering (specializing in otolaryngology), PET manufacturing, and acoustic engineering. Since 2020, he has been a professor at National Formosa University (NFU).

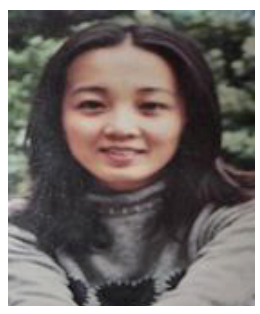

Hui-Fang Lin received her B.S. and M.S. degrees from NFU in 2014 and 2016, respectively. She is currently a Ph.D. student. Her research interest is in $\mathrm{CFD}$. 